\title{
Clavine Alkaloids as Precursors of Peptide-type Ergot Alkaloids*
}

\author{
STIG AGURELL \\ Royal Pharmaceutical Institute, Stockholm, Sweden \\ MARTIN JOHANSSON \\ Institute of Physiological Botany, University of Uppsala, Uppsala, Sweden

\begin{abstract}
The relations of some clavine alkaloids to the biosynthesis of lysergic acid type ergot alkaloids has been investigated in a strain of Claviceps purpurea. Agroclavine and elymoclavine were found to serve as precursors of lysergic acid derivatives whereas the found biogenetic sequence lysergene $\rightarrow$ lysergol $\rightarrow$ penniclavine apparently was unrelated to the biosynthesis of the peptide type ergot alkaloids.
\end{abstract}

The ergot alkaloids comprise some thirty ergoline derivatives produced by 1 fungi belonging to Claviceps ${ }^{1}$ and Aspergillus ${ }^{2}$ but also by higher plants. ${ }^{3}$ These alkaloids may be divided into two groups: one group a series of simpler ergoline derivatives, the clavine series; the other group may be considered as derivatives of lysergic acid joined in amide linkage with a sidechain. The biogenetic problems associated with the ergot alkaloids have lately received much attention and the biosynthesis of the ergoline skeleton ${ }^{4}$ as well as the biogenetic interrelations of a number of clavine alkaloids ${ }^{4,5}$ have been investigated. Recent data ${ }^{6,7}$ support the view that 4-dimethylallyltryptophan is an intermediate on the biosynthetic path from the primary precursors mevalonic acid and tryptophan to the final ergoline skeleton.

The biogenetic relations between the clavine type and the lysergic acid type ergot alkaloids has been the object of numerous speculations. Some authors ${ }^{4}$ assume an oxidation of the C-methyl group of agroclavine via elymoclavine to lysergic acid, while others ${ }^{1,4}$ suggest that the two groups may be formed by different pathways from a common precursor such as lysergic aldehyde. Also other theories have been put forth.4,8 In connection with our earlier work on the in vivo oxidation of agroclavine (I) to elymoclavine

* Presented at Nordiskt Naturproduktssymposium, Abisko, Sweden, August 20-25, 1964.

Acta Chem. Scand. 18 (1964) No. 10 
(II) in a clavine type ergot strain, we obtained indications of a further oxidation of the latter compound to lysergic acid ${ }^{9}$ (III). Efficient incorporation of elymoclavine into the lysergic acid derivatives ergotamine $(I V, R=$ tricyclic peptide) and lysergic acid amide (IV, $\mathrm{R}=\mathrm{H}$ ) has recently been demonstrated by Mothes, Gröger et al.10,11

The purpose of this investigation was to test the ability of some clavine alkaloids to act as precursors of the lysergic acid moiety of ergotamine. The tested precursor alkaloids were chosen on the basis of previous experiences, ${ }^{5,10}$ had been suggested as possible precursors ${ }^{8}$ or were possible predecessors due to their stereochemical configuration. The possible conversion of agroclavine, elymoclavine, lysergol, lysergene, penniclavine and isolysergol to the lysergic acid derivatives ergotamine, ergotaminine and ergometrine was studied.

\section{EXPERIMENTAL}

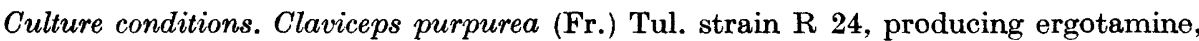
ergotaminine, chanoclavine and small amounts of ergometrine, ${ }^{12}$ was cultivated at $25^{\circ}$ on our standard medium containing $120 \mathrm{mg} / \mathrm{l}$ of oxine. ${ }^{13}$ The flasks, each containing $20 \mathrm{ml}$ medium, were inoculated with single pieces of mycelium which were allowed to develop into sclerotia-like structures for 12-15 days, at which time the mycelia weighed 3.8$5.2 \mathrm{~g}$ (wet weight) and contained $0.1-0.3 \mathrm{mg}$ of alkaloids.

Chromatography. System FBP: formamide-treated Whatman 3MM paper developed with benzene-pyridine $(6: 1)$ as solvent. ${ }^{14}$ System FCCB: formamide-treated Whatman $3 \mathrm{MM}$ paper with a solvent consisting of carbon tetrachloride-chloroform-benzene $(7: 2: 1){ }^{15}$ System FN: formamide-treated paper with carbon tetrachloride-chloroform-benzene (3:4:1). Thin layer chromatography on Silica Gel G coated glass plates were performed with following solvents: MC, methanol-chloroform (2:8);15 EED, ethyl acetate-ethanoldimethylformamide (13:1:1); ${ }^{14} \mathrm{DC}$, diethylamine-chloroform $(1: 9)$.

Mixtures of labeled alkaloids were separated on formamide treated papers in FBP ${ }^{14}$ and FCCB. ${ }^{15}$ Ergotamine, agroclavine, lysergene and ergotaminine were separated on formamide treated Whatman 3MM paper first with carbon tetrachloride-chloroformbenzene (FN) and then followed by benzene-pyridine until the front reached ergotamine. Alkaloids were located with Ehrlich's reagent and by fluorescence under UV.light.14

Chromatographic identification ${ }^{14}$ of lysergic acid was made after basic hydrolysis of the alkaloids in a capillary tube for $2 \mathrm{~h}$ at $100^{\circ}$ with $5 \% \mathrm{KOH}$ in $50 \%$ aqueous methanol. Acid hydrolysis of ergotamine and chromatographic identification of the amino acids formed from the tricyclic peptide moiety was performed as described by Genest and Farmilio. ${ }^{16}$

Labeled alkaloids. ${ }^{14} \mathrm{C}$-labeled clavine alkaloids were produced from mevalonic acid-2- ${ }^{14} \mathrm{C}$ as described previously. ${ }^{5}$ The purified mixture of clavine alkaloids was separated by paper chromatography in system FBP and the individual alkaloids further purified by TLC with methanol-chloroform. Elymoclavine was crystallized with carrier, m.p. $240-245^{\circ}$ (not corr.), as was also agroclavine, m.p. 197-200 (not corr.). Labeled lysergol, isolysergol and lysergene were prepared from elymoclavine ${ }^{14} \mathrm{C} .5$ The purity of the individual alkaloids were determined by chromatography in FBP, DC, and MC.

Conversion experiments. After an incubation time of 12-15 days the medium was removed aseptically from the flasks. Filter-sterilized solutions $(1-1.5 \mathrm{ml} /$ flask, phosphate buffer $\mathrm{pH}$ 6.5) of the labeled compounds were sprayed on the lower surface of the mycelia. After another 6-8 days the mycelia were individually thoroughly ground with sand and extracted with portions of $1 \%$ aqueous acetic acid, ammonia added, and the alkaloids extracted repeatedly with chloroform. The concentrated alkaloidal extract was purified further by transfer to acid and then back into chloroform after addition of ammonia to the acid extract. ${ }^{14}$

Assay procedures. Aliquots of the recovered radioactive alkaloid mixture were separated on formamide paper in a suitable system and the activities of the different compounds 
were determined in a Nuclear Chicago $4 \pi$ Actigraph II Model 1032 chromatogram scanner. One half of the scanned chromatogram was treated with Ehrlich's reagent to locate the non-fluorescent alkaloids, the other half was used for quantitative determination ${ }^{5}$ with van Urk's reagent. Standard curves were prepared with corresponding reference compounds and the absorbance measured spectrophotometrically (Beckman DU) at $380 \mathrm{~m} \mu$ for lysergene, at $400 \mathrm{~m} \mu$ for penniclavine and at $550 \mathrm{~m} \mu$ for the remaining alkaloids.

Radioactivities were also determined in a Packard Tri-Carb Liquid Scintillation Spectrometer Model 314 AS in a scintillator solution (TPP) of toluene, PPO $0.5 \%$, dimethyl-POPOP (Packard Instrument Co.) $0.03 \%$. Aqueous solutions were measured in a scintillator (HETPP) consisting of $14 \mathrm{ml}$ TPP, $2.0 \mathrm{ml}$ absolute ethanol and $0.10 \mathrm{ml}$ $\mathrm{H}_{2} \mathrm{O}$ or in XDC scintillator. ${ }^{17}$ The counting efficiences for ${ }^{14} \mathrm{C}$ in the different systems were approximately: in TPP $70 \%$, in HETPP $72 \%$ and in XDC $\left(6.7 \% \mathrm{H}_{2} \mathrm{O}\right) 63 \%$. All count rates were calculated by the internal standard method as absolute count rates, disintegrations per minute (d.p.m.).

\section{RESULTS}

Identification of alkaloids. The previous identification ${ }^{12}$ of the alkaloids produced by ergot strain $R 24$ was further verified by separating in MC the alkaloidal mixture from a number of cultures. The eluted individual alkaloids were then chromatographed in systems MC, DC, EED, and FBP and found to coincide with reference compounds. Alkaline hydrolysis of ergometrine and ergotamine gave chromatographic evidence of formation of lysergic acid and hydrolysis of ergotamine with $\mathrm{HCl}^{16}$ gave the expected two amino acids, proline and phenylalanine.

Conversion experiments. Preliminary experiments with strain $\mathrm{R} 24$ showed that elymoclavine- ${ }^{14} \mathrm{C}$ (II) or lysergol- ${ }^{14} \mathrm{C}$ (VI) introduced into the medium of 14 days old cultures were not significantly incorporated into ergotamine (IV) or metabolized to yield any other alkaloid. This approach had previously been used in our work with clavine producing strains. ${ }^{5}$ We therefore tested incorporation of elymoclavine- ${ }^{14} \mathrm{C}$ applied directly to the mycelium after removal of the medium, similarly as described by Winkler and Mothes. ${ }^{18}$ Tests carried out after various treatments of the mycelia (freeze-dried, dried ${ }^{18}$ or wet, whole or broken) at different $\mathrm{pH}$ indicated that whole, wet or dried, mycelia at $\mathrm{pH} 5-7$ showed a satisfactory conversion of elymoclavine to ergotamine (data not recorded here). For convenience, the conditions defined under Experimental were used.

The results obtained with labeled agroclavine (I) and elymoclavine (II) are tabulated in Table 1. In this and in the following table, the presented figures represent the average per culture flask in the particular experiment. Although different cultures in the same experiment showed the same qualitative picture, the quantitative differences were sometimes considerable. The quotation marks around "elymoclavine" and "lysergol" in Tables 1 and 2 are used because the utilized chromatographic systems do not clearly distinguish between these two alkaloids (Fig. 2). Some distinction can be made due to the fluorescence of lysergol under UV-light and the darker color it produces with Ehrlich's reagent. A chromatogram scan of metabolized elymoclavine- ${ }^{14} \mathrm{C}$ is presented in Fig. 1. As Table 1 illustrates, both agroclavine and elymoclavine are utilized for the biosynthesis of ergotamine, ergotaminine and ergometrine. Further, elymoclavine serves as precursor of penniclavine and similarly

Acta Chem. Scand. 18 (1964) No. 10 


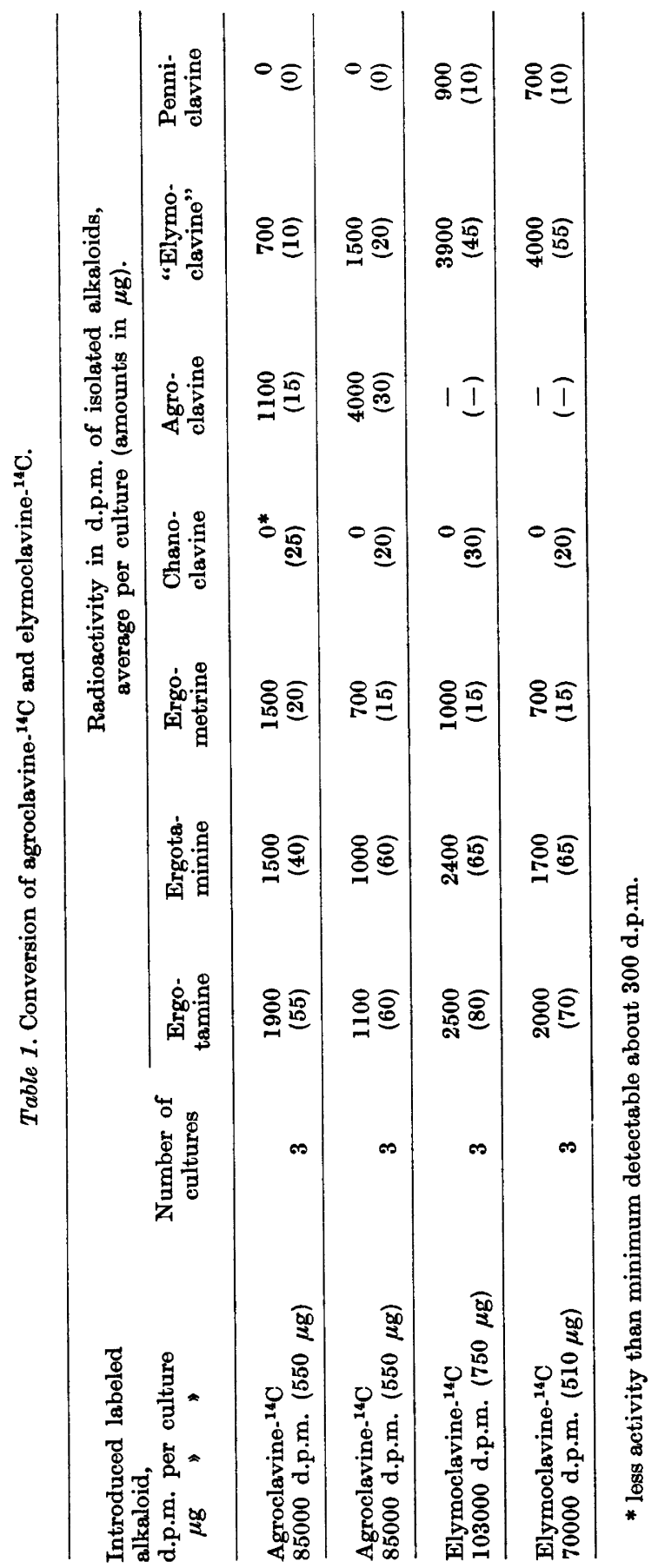

Acta Chem. Scand. 18 (1964) No. 10 


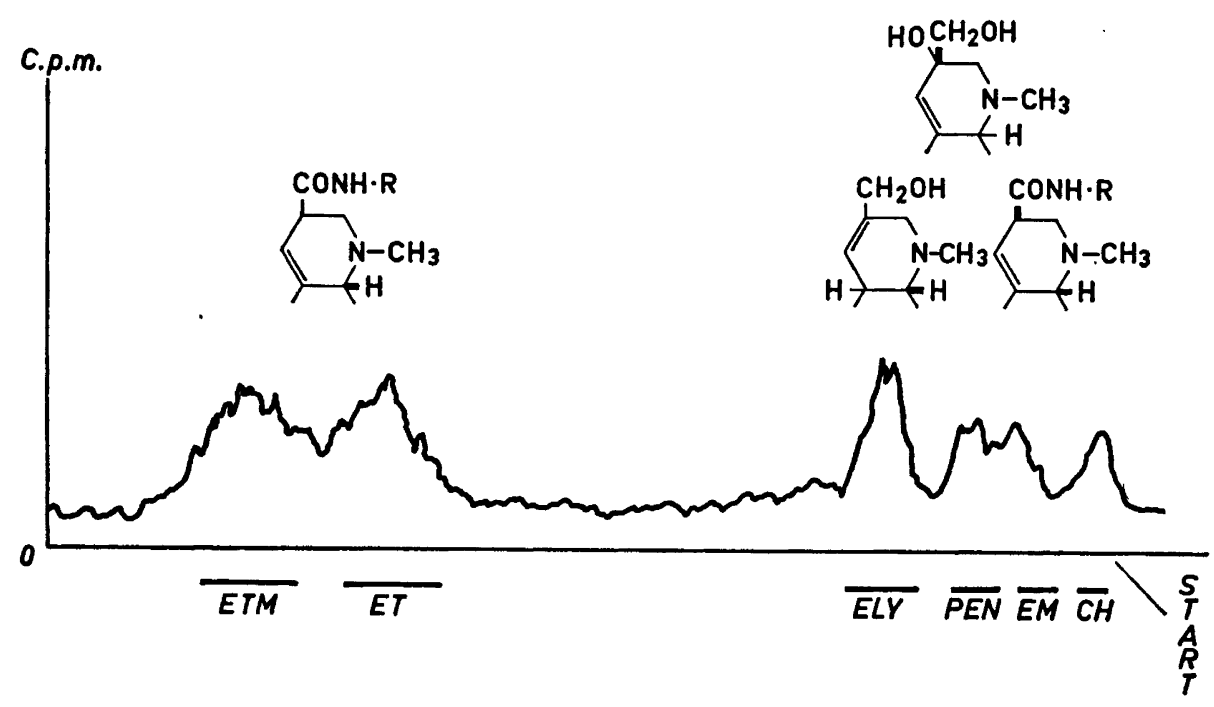

Fig. 1. Chromatogram scan of metabolized elymoclavine- ${ }^{14} \mathrm{C}$. Abbreviations: ETM = ergotaminine, $\mathbf{E T}=$ ergotamine, AGRO = agroclavine, $\mathrm{LEN}=$ lysergene, $\mathrm{LYL}=$ lysergol, ELY = elymoclavine, PEN = penniclavine, EM = ergometrine, $\mathrm{CH}=$ chanoclavine.

agroclavine was converted to a minor degree to setoclavine (data not given in the table). In two sets of experiments not tabulated here, where comparatively large amounts $\left(2-5 \mathrm{mg} /\right.$ flask) of agroclavine- ${ }^{14} \mathrm{C}$ were fed, there was little metabolism of this compound to anything but a small conversion to elymoclavine. The recovery of agroclavine was 60-80\% compared to a few per cent in the experiments reported in Table 1.

Table 2 shows the experimental results obtained with lysergene, lysergol and penniclavine, together with an experiment involving isolysergol, the C-8 isomer of lysergol. As pointed out above, no clear-cut separation between lysergol and elymoclavine in alkaloid mixtures could be made. However, the rather exclusive labeling of lysergol seems likely on account of the similar results with lysergene and lysergol and the lack of transfer of label from these alkaloids to, e.g., ergotamine. Chromatogram scans of metabolized lysergene- ${ }^{14} \mathrm{C}$ and lysergol- ${ }^{14} \mathrm{C}$ are shown in Fig. 2. As appears from Table 2 and Fig. 2 lysergene- ${ }^{14} \mathrm{C}$ rendered lysergol and penniclavine radioactive and lysergol was a precursor of penniclavine whereas penniclavine was metabolically rather inert.

\section{DISCUSSION}

It was earlier found that elymoclavine is used efficiently for the biosynthesis of ergotamine in a Claviceps strain ${ }^{10}$ and this result was confirmed in our experiments. We had earlier found an oxidation of agroclavine to elymoclavine 


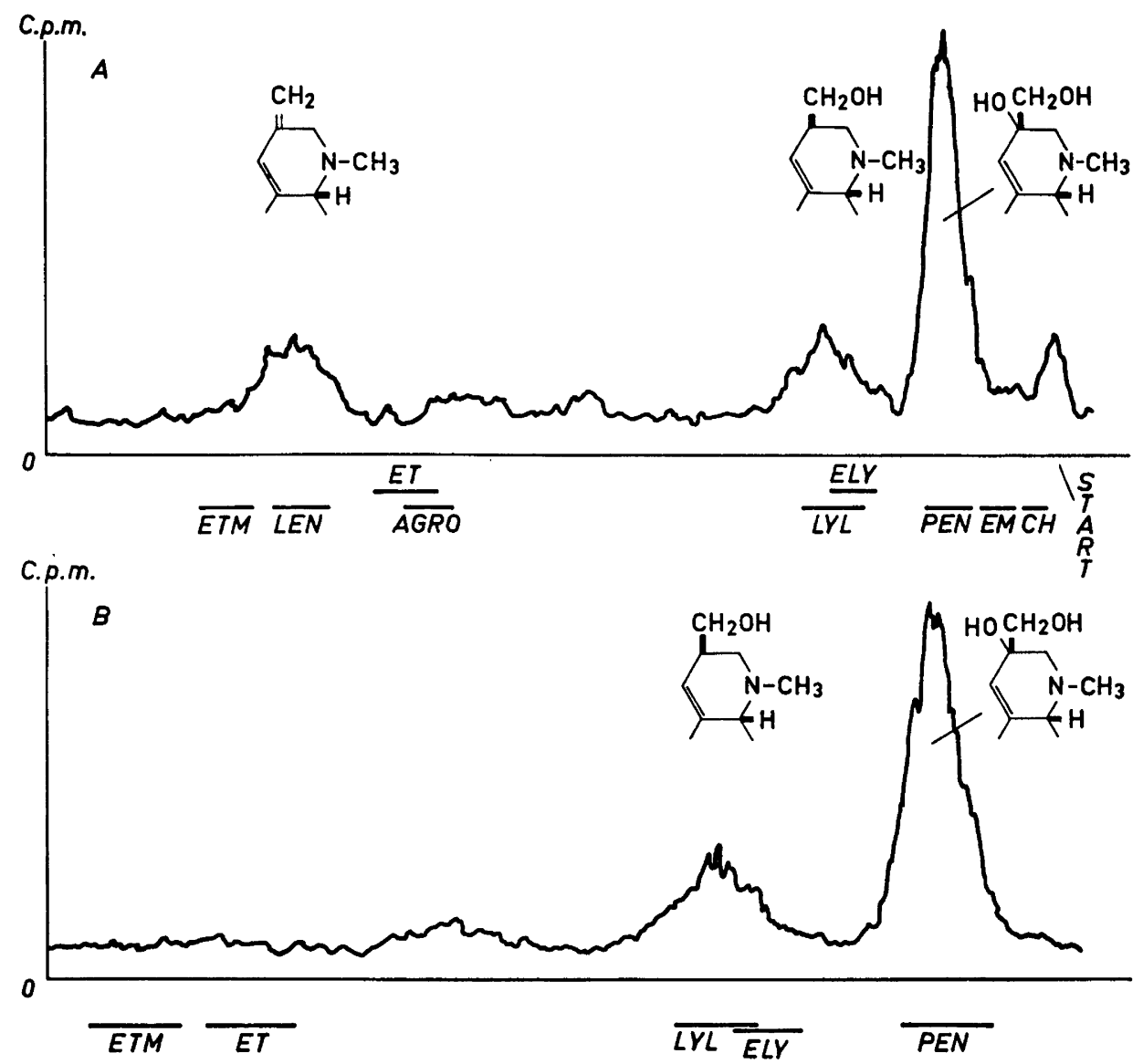

Fig. 2. Chromatogram scan of metabolized A. lysergene- ${ }^{14} \mathrm{C}$ B. lysergol- ${ }^{14} \mathrm{C}$. Abbreviations as in Fig. 1.

in a clavine producing strain ${ }^{5}$ and the same reaction seemed to occur in strain $\mathrm{R} 24$, where added agroclavine- ${ }^{14} \mathrm{C}$ transferred label to elymoclavine as well as some lysergic acid derivatives viz. ergotamine, ergotaminine, and ergometrine. One would assume that agroclavine (I) was transformed to elymoclavine (II) which, in turn, was used for the biosynthesis of the lysergic acid (III) part of the peptide alkaloids (IV) (Fig. 3). Calculated from the figures in Table 1, 29-55\% of the ergotamine of the cultures was derived from the introduced clavine precursors. The loss of radioactivity from the alkaloidal fraction is quite striking in Tables 1 and 2, but the high specific activities of the derived alkaloids, as compared to their labeled precursors, excludes the possibility of incorporation of radioactivity into new-formed alkaloids from labeled break-down fragments. This possibility is also unlikely when one 


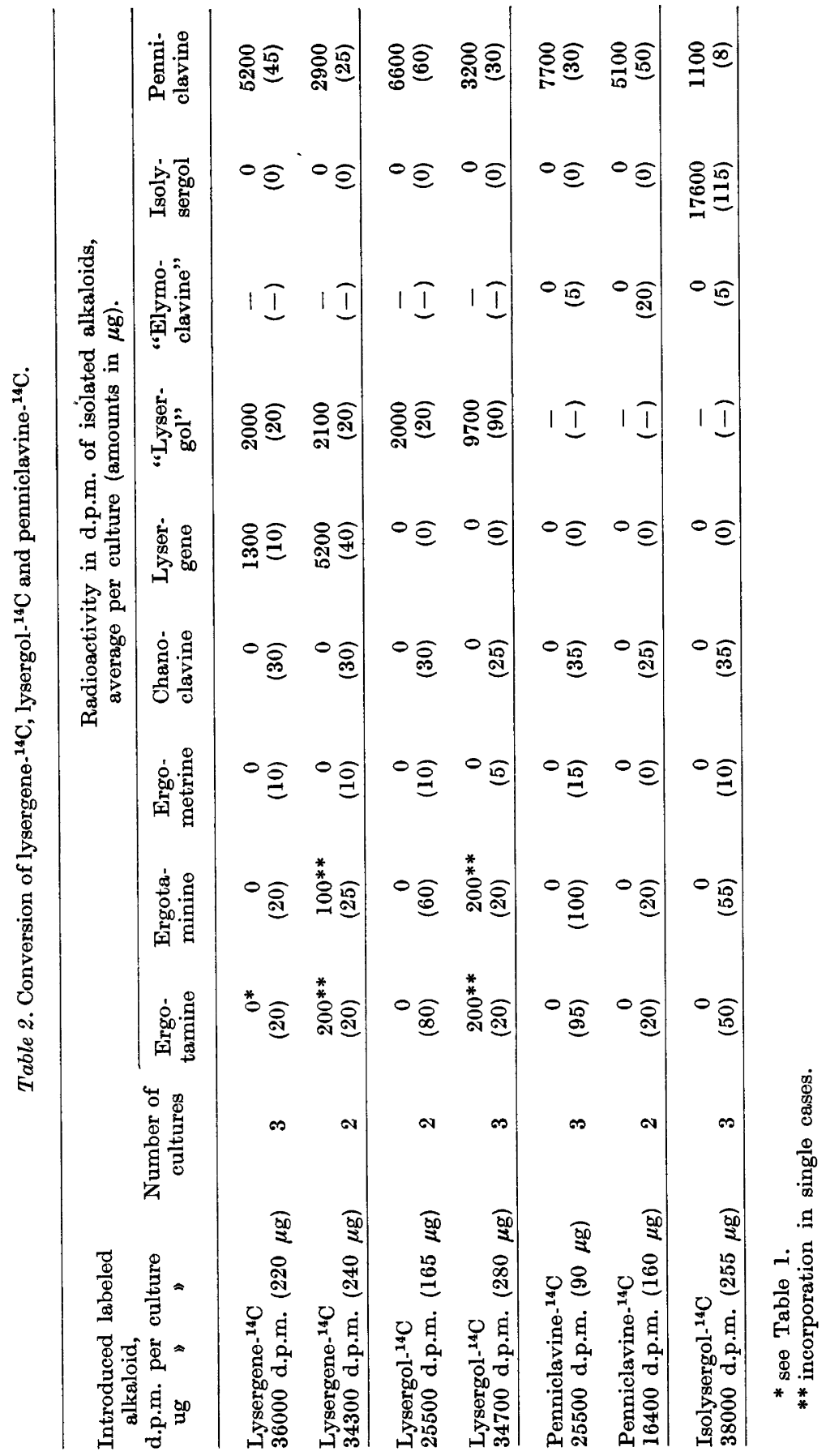

Acta Chem. Scand. 18 (1964) No. 10 
considers the different labeling patterns depending on which labeled precursor that is introduced. Gröger has found direct evidence ${ }^{\mathbf{1 1}}$ for a non-scrambling of label during conversion. The profound instability of the alkaloids noted here, is partly due to chemical destruction ${ }^{13}$ and has previously been observed by us ${ }^{5}$ and others. ${ }^{18}$

Earlier we ${ }^{5}$ and Baxter et al. ${ }^{19}$ found chanoclavine added to the medium of two clavine producing strains to be inactive as a precursor, whereas Abe ${ }^{8}$ under some not further defined conditions found a reversible reaction agroclavine $\rightleftharpoons$ chanoclavine. Under the conditions described above chanoclavine did not become significantly labeled (Tables 1 and 2) from the tested clavine alkaloids. A more prolonged exposure, which Mothes and Winkler ${ }^{20}$ found necessary, might possibly have led to transfer of label from elymoclavine to chanoclavine. As suggested by Weygand, ${ }^{4}$ this may be an alternative reaction. The conversion, although very scanty, of isolysergol to penniclavine (Table 2) having the opposite configuration at C-8, rather than isopenniclavine, seems unexpected but might /be explained by an oxidation at C-8 of isolysergol forming a planar ionic or free radical intermediate.

$\mathrm{Abe}^{8}$ has assumed the existence of a cyclic path in microorganisms: elymoclavine $\rightarrow$ lysergol $\rightarrow$ lysergene $\rightarrow$ agroclavine $\rightarrow$ elymoclavine. Such a proposed cycle does not seem to operate under our conditions since radioactivity from labeled lysergol and lysergene did not enter the lysergic acid derivatives which, as shown, derive from agroclavine and elymoclavine. Instead, a reaction sequence (Fig. 3 ): lysergene (V) $\rightarrow$ lysergol (VI) $\rightarrow$ penniclavine (VII) apparently not related to the peptide alkaloids, was found. This path is similar to the results earlier obtained with lysergene in a clavine alkaloid producing strain, ${ }^{5}$ although at that time, possibly due to experimental or
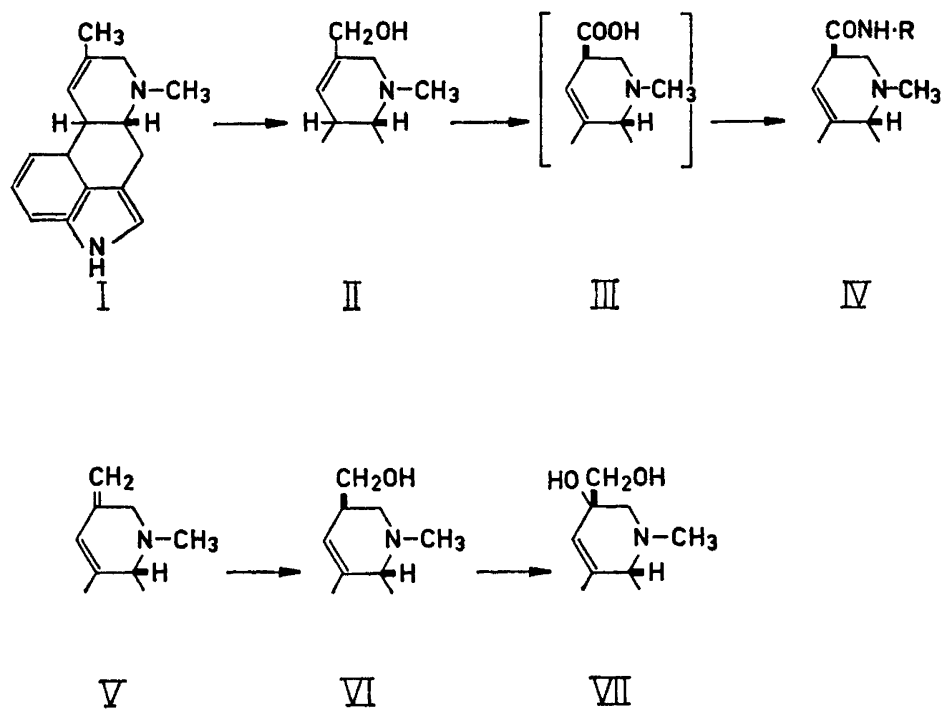

Fig. 3. Probable biosynthetic paths found in C. purpurea strain R 24. 
strain differences, no significant metabolism of lysergol was found. The discrepancies ${ }^{19}$ between our experimenta findings and Abe's hypothesis may possibly be explained by assuming that the added alkaloids cannot reach the proper enzymatic sites or are metabolized before they reach them. This is however unlikely since it does not hold true for, e.g., elymoclavine. Also, none of the alkaloids in the sequence V-VII in Fig. 3 is detectable in control cultures. Another explanation may be that lysergene seemingly depresses the formation or increases the destruction of ergotamine and ergotaminine since the quantities of these alkaloids were low in lysergene fed cultures. This was also found in a set of cultures, each fed $200 \mu \mathrm{g}$ of non-labeled lysergene and where the average ergotamine content was about one half of that of the control.

Acknowledgements. This study was supported by a grant from the Swedish Natural Science Research Council. A grant from the Royal Pharmaceutical Institute towards the purchase of a scintillation counter is also gratefully acknowledged.

\section{REFERENCES}

1. Abe, M. A Consideration concerning the Biosynthesis of the Ergot Alkaloids 2. Arbeitstagung "Biochemie und Physiologie der Alkaloide" Halle (Saale) May 21-25, 1960.

2. Spilsbury, J. F. and Wilkinson, S. J. Chem. Soc. 19612085.

3. Hofmann, A. Planta Medica 9 (1961) 354.

4. For recent reviews see: Winkler, K. and Gröger, D. Pharmazie 17 (1962) 658; Weygand, F. and Floss, H.-G. Angew. Chem., Intern. Ed. 2 (1963) 243; Ramstad, E. and Agurell, S. Ann. Rev. Plant Physiol. 15 (1964) 143.

5. Agurell, S. and Ramstad, E. Arch. Biochem. Biophys. 98 (1962) 457.

6. Plieninger, H., Fischer, R. and Liede, V. Ann. 672 (1964) 223.

7. Weygand, F., Floss, H.-G., Mothes, U., Gröger, D. and Mothes, K. Z. Naturforsch. $19 b$ (1964) 202.

8. Abe, M., Yamatodani, S., Yamono, T., Kozu, Y. and Yamada, S. Agr. Biol. Chem. (Tokyo) 27 (1963) 659.

9. Agurell, S. and Ramstad, E. Tetrahedron Letters 1962501.

10. Mothes, K., Winkler, K., Gröger, D., Floss, H.-G., Mothes, U. and Weygand, F. Tetrahedron Letters 1962933.

11. Gröger, D., Schütte, H. R. and Stolle, K. Z. Naturforsch. $18 b$ (1963) 1850.

12. Johansson, M. Physiol. Plantarum 17 (1964) 507.

13. Johansson, M. Symbolae Botan. Upsalienses 17 (1962) 2.

14. Agurell, S. and Ramstad, E. Lloydia 25 (1962) 67.

15. Agurell, S., Ramstad, E. and Ullstrup, A. J. Planta Medica 11 (1963) 392.

16. Genest, K. and Farmilio, C. G. J. Pharm. Pharmacol. 16 (1964) 250.

17. Bruno, G. A. and Christian, J. E. Anal. Chem. 33 (1961) 1216.

18. Winkler, K. and Mothes, K. Planta Medica 10 (1962) 208.

19. Baxter, R. M., Kandel, S. I., Okany, A. and Tam, K. L. J. Am. Chem. Soc. 84 (1962) 4350 .

20. Winkler, K. and Mothes, K. Tetrahedron Letters 19621243.

Received September 7, 1964. 